\title{
Social Determinants of Health and Risk of SARS-CoV-2 Infection in Community-Dwelling Older Adults Living in a Rural Latin American Setting
}

\author{
Oscar H. Del Brutto ${ }^{1,4}\left(\right.$ D $\cdot$ Robertino M. Mera ${ }^{2} \cdot$ Bettsy Y. Recalde ${ }^{3} \cdot$ Aldo F. Costa $^{3}$
}

Published online: 15 July 2020

○) Springer Science+Business Media, LLC, part of Springer Nature 2020

\begin{abstract}
High social risk, as measured by the social determinants of health (SDH), may increase the risk of SARS-CoV-2 infection. However, this association has not been studied in rural communities. Using the Atahualpa Project cohort, we aimed to assess the association between SDH and SARS-CoV-2 seropositivity in community-dwelling older adults living in rural Ecuador. SARS-CoV-2 antibodies were determined in 319 individuals aged $\geq 60$ years that completed a validated field instrument to assess their social risk before the introduction of this novel pandemic. Multivariate models were fitted to assess the independent association between SDH—and each of their components—and SARS-CoV-2 seropositivity, after adjusting for relevant covariates. According to the Gijon scale, 102 (32\%) individuals had a high social risk ( $\geq 10$ points). A total of 141 (44\%) individuals were seropositive to SARS-CoV-2. A fully-adjusted logistic regression model showed an independent) association between social risk and SARS-CoV-2 positivity (OR 1.15; 95\% CI 1.04-1.27; $p=0.008$ ). For every unit of the total SDH score, the odds of SARS-CoV-2 seropositivity increased 15\% (95\% CI 3.7-27\%). In addition, multivariate models showed that the individual component of SDH more strongly associated with SARS-CoV-2 seropositivity was housing, which suggested that lack of basic home facilities may increase the risk of SARS-CoV-2 infection. Knowledge on the association between high social risk and SARS-CoV-2 infection is indispensable for the development of cost-effective preventive strategies for controlling modifiable factors that are in the path of SARS-CoV-2 infection among older adults living in underserved communities.
\end{abstract}

Keywords Social determinants of health $\cdot$ SARS-CoV-2 $\cdot$ Older adults $\cdot$ Rural communities

\section{Introduction}

The novel Coronavirus Disease 2019 (COVID-19) global pandemic, caused by the Coronavirus 2 Severe Acute Respiratory Syndrome (SARS-CoV-2), has affected more than 10 million people and claimed the lives of more than 500

Oscar H. Del Brutto

oscardelbrutto@hotmail.com

1 School of Medicine, Universidad Espíritu Santo - Ecuador, Samborondón, Ecuador

2 Department of Epidemiology, Gilead Sciences, Inc., Foster City, CA, USA

3 Community Center, The Atahualpa Project, Atahualpa, Ecuador

4 Air Center 3542, PO Box 522970, Miami, FL 33152-2970, USA thousand persons [1]. Highly prevalent in urban centers of China, USA, and several European countries, the disease has spread to Africa and Latin America as well [2-4]. People living in underserved rural communities of these countries are likely to be especially vulnerable to this pandemic because of multiple factors inherent to under-development [5-7]. Despite the vast information on SARS-CoV-2 prevalence and associated risk factors from large urban centers, there is little or nil evidence of the burden and profile of individuals with SARS-CoV-2 in remote rural communities. Several editorial comments have suggested that disparities in the social determinants of health (SDH) may influence SARS-CoV-2 acquisition and severity in these settings [8-10], however, there are no data supporting this hypothesis. Taking the unique opportunity of the Atahualpa Project, where SDH had been measured a few months before the start of the novel COVID-19 pandemic [11], we aimed to assess the relevance of the different 
components of the SDH on SARS-CoV-2 infection in a rural Ecuadorian village (Atahualpa), where this virus is highly prevalent [manuscript under review elsewhere].

\section{Methods}

\section{Study Population}

The Atahualpa Project is a population-based prospective cohort study designed to investigate factors responsible for the increasing burden of neurological and cardiovascular diseases in rural Ecuador [12]. Atahualpa is representative of rural villages of Coastal Ecuador [13]. The population is homogeneous regarding race/ethnicity, lifestyles and diet. The wheather is hot and dry, with 12 daily hours of sunlight year-round. The village has electricity and almost all houses have piped water. However, a sizable proportion of houses do not have flushing toilets and closed sewage disposal systems. Many streets are not paved and several houses are made of cane or wood. Atahualpa residents are Ecuadorian natives with little evidence of cross-breeding (Amerindians). Almost all men belong to the blue-collar class and most women are homemakers. Atahualpa has a very low index of migration (less than 30 adults leave the village every year) and the adherence of the population to the Atahualpa Project has been high, which makes it an optimal setting for conducting population-based studies [14]. The village has only one health center of the Minister of Health (ambulatory medical care), and the nearest hospital is located about 10 miles away in a small city (Ancón).

\section{Study Design}

We retrieved data on SDH obtained from Atahualpa residents aged $\geq 60$ years. Such data was prospectively collected by means of a door-to-door survey conducted in November and December 2019, as part of a study attempting to assess correlates of dementia in rural Ecuador [11]. Information on SDH was evaluated in the light of the results of a doorto-door serological survey aimed to assess the presence of antibodies to SARS-CoV-2 in the same population (study conducted on May, 2020). Multivariate models were fitted to assess the independent association between SDH-and each of their components-and SARS-CoV-2 seropositivity, after adjusting for relevant covariates (see below). The study and the informed consent forms-signed by all participants before enrollment-were approved by the I.R.B. of Universidad de Especialidades Espiritu Santo, Ecuador (IORG: 0010320; FWA: 00028878).

\section{Social Determinants of Health}

The Gijon Scale was used to measure SDH. This reliableand validated-field instrument evaluates five components of risk situations and social problems, including family situation, economic status, housing, social relationships and support networks [15]. Each component is weighted on a 1 to 5 scale, for a maximal score of 25 , with a score of $<10$ points indicating low social risk. The Gijon scale was used because it has been officially endorsed by the Minister of Public Health of Ecuador, and is applicable to the living conditions of the study population.

\section{Serological Tests}

Detection of SARS-CoV-2 IgM and IgG antibodies was performed using the BIOHIT SARS-CoV-2 antibody test kit, colloidal gold method (BIOHIT Health Care Ltd., Cheshire, UK). The manufacturer reports $97.5 \%$ sensitivity with $99.5 \%$ specificity for $\operatorname{IgM}$, and $97.5 \%$ sensitivity with $100 \%$ specificity for IgG detection of this kit. In this immunochromatrogaphic test, immunoglobulins from the sample react with SARS-CoV-2 recombinant antigens linked to colloidal gold particles and the resulting antigen-antibody complexes migrate along the membrane and react with mouse anti-human IgG or mouse anti-human IgM anti-antibodies. Following manufacturer instructions, $10 \mu \mathrm{l}$ of capillary blood together with $80 \mu \mathrm{l}$ of a buffer solution were applied to the test card's hole and after $15 \mathrm{~min}$, purplish red color lines developed at the control line (mandatory) and at the $\mathrm{IgG}$ and/or IgM lines (in positive cases). Digital photos were taken at the field and independently read by two of the authors blinded to any other information. Discrepancies were resolved by consensus.

\section{Covariates of Interest}

Covariates were selected if they have been suggested to play a role in SARS-CoV-2 acquisition or spread [16], and included age, gender, level of education (primary school education or higher), alcohol intake (dichotomized in $\leq 50$ or $>50 \mathrm{~g} /$ day), and history of cardiovascular risk factors, according to criteria proposed by the American Heart Association [17]. These data were extracted from the archives of the Atahualpa Project and updated during the house visits.

While preventive measures are considered essential to reduce SARS-CoV-2 acquisition and spread [18], they were not included as covariates because they were not taken into account by most study participants. More than $90 \%$ of the population used face masks; however, the same mask was frequently used for more than two weeks (because of costs) 
and masks were not properly used (often below the nostrils). Frequent hand washing with soap or alcohol was not a common practice, nor the use of surface disinfectants. Social distancing was not taken into account, however, the Ecuadorian government prohibited social meetings of more than 10 persons, closed bars and restaurants in the village, and imposed a curfew (from 2 p.m. to 5 a.m.) since the first week of April. Likewise, some household factors that have also been associated with SARS-CoV-2 acquisition and spread (in-house crowding, lack of flushing toilets) were not considered as covariates because of collinearity, since housing characteristics are part of the SDH.

\section{Statistical Analysis}

Data analyses were carried out by using STATA version 16 (College Station, TX, USA). In univariate analyses, continuous variables were compared by linear models and categorical variables by the $x^{2}$ or Fisher exact test, as appropriate. Logistic regression models were fitted to assess the independent association between SDH (and their different components) and SARS-CoV-2 seropositivity (as the dependent variable), after adjusting for the above-mentioned covariates.

\section{Results}

From 352 individuals aged $\geq 60$ years that were actively enrolled in the Atahualpa Project as of May 31, 2019, 27 had either died $(n=20)$ or emigrated $(n=7)$ in the past 12 months. Thus, 325 older adults were living in the village as of May 25, 2020, and from these, six declined participation in the current survey (coverage 98.2\%).

The mean age of the 319 participants was $70.5 \pm 7.8$ years (median age: 69 years), 187 (59\%) were female, 240 (75\%) had primary school education only, and $52(16 \%)$ disclosed heavy alcohol intake. Twelve individuals (4\%) were current smokers, 74 (23\%) had a body mass index $\geq 30 \mathrm{~kg} / \mathrm{m}^{2}, 21$ (7\%) had poor physical activity, $13(4 \%)$ had a poor diet, 134 (42\%) had blood pressure $\geq 140 / 90 \mathrm{mmHg}, 92$ (29\%) had fasting glucose $\geq 126 \mathrm{mg} / \mathrm{dl}$, and 45 (14\%) had total cholesterol levels $\geq 240 \mathrm{mg} / \mathrm{dl}$.

According to the SDH, 102 (32\%) individuals had a high social risk ( $\geq 10$ points in the Gijon scale), and the remaining $217(68 \%)$ had a low social risk. The mean score in the Gijon scale was $9.03 \pm 2.5$. A total of 141 (44\%) individuals were seropositive to SARS-CoV-2 (including 117 who were reactive to both $\operatorname{IgM}$ and $\operatorname{IgG}$, seven to only $\operatorname{IgM}$, and 17 to only IgG). As IgM and IgG responses in SARS-CoV-2 develop with only a few days of difference [19], we defined seropositivity as a positive response to any of them. The Kappa coefficient for interrater agreement was 0.91 (95\% CI 0.87-0.96) for seropositivity to SARS-Cov-2.

Table 1 depicts the characteristics of participants across categories of social risk and SARS-CoV-2 serological status (univariate analyses). Individuals with high social risk were older, more often female, and had more often primary school education only, worse physical activity and poor diet, than those with low social risk. In contrast, the single covariate significantly associated with SARS-CoV-2 seropositivity was hypercholesterolemia. Also in univariate analysis, the mean $( \pm \mathrm{SD})$ total score in the Gijon scale was higher in the

Table 1 Characteristics of Atahualpa residents aged $\geq 60$ years across categories of social risk and SARS-CoV-2 serological status (univariate analyses)

\begin{tabular}{|c|c|c|c|c|c|c|c|}
\hline \multirow[t]{2}{*}{ Variable } & \multirow[t]{2}{*}{ Total series $(\mathrm{n}=319)$} & \multicolumn{3}{|c|}{ Social determinants of health } & \multicolumn{3}{|c|}{ SARS-CoV-2 serological status } \\
\hline & & $\begin{array}{l}\text { Low } \\
\text { social risk } \\
(\mathrm{n}=217)\end{array}$ & $\begin{array}{l}\text { High } \\
\text { social risk } \\
(\mathrm{n}=102)\end{array}$ & $p$ value & $\begin{array}{l}\text { Seron- } \\
\text { egative } \\
(\mathrm{n}=178)\end{array}$ & $\begin{array}{l}\text { Sero- } \\
\text { positive } \\
(\mathrm{n}=141)\end{array}$ & $p$ value \\
\hline Age, years $($ mean $\pm S D)$ & $70.5 \pm 7.8$ & $69.2 \pm 6.8$ & $73.4 \pm 9$ & $<0.001 *$ & $70.9 \pm 8.4$ & $70 \pm 7.1$ & 0.310 \\
\hline Female, n (\%) & 187 (59) & $118(54)$ & $69(68)$ & $0.025^{*}$ & $103(58)$ & $84(60)$ & 0.7548 \\
\hline Primary school education, n (\%) & $240(75)$ & $152(70)$ & $88(86)$ & $0.002 *$ & $128(72)$ & $112(79)$ & 0.122 \\
\hline Heavy alcohol intake, n (\%) & $52(16)$ & $37(17)$ & $15(15)$ & 0.597 & $28(16)$ & $24(17)$ & 0.757 \\
\hline Current smoker, n (\%) & $12(4)$ & $9(4)$ & $3(3)$ & 0.597 & $7(4)$ & $5(4)$ & 0.857 \\
\hline Body mass index $\geq 30 \mathrm{~kg} / \mathrm{m}^{2}, \mathrm{n}(\%)$ & $74(23)$ & $53(24)$ & $21(21)$ & 0.449 & $40(22)$ & $34(24)$ & 0.730 \\
\hline Poor physical activity, n (\%) & $21(7)$ & $8(4)$ & $13(13)$ & $0.002^{*}$ & $16(9)$ & $5(4)$ & 0.052 \\
\hline Poor diet, n (\%) & $13(4)$ & $4(2)$ & $9(9)$ & $<0.001 *$ & $9(5)$ & $4(3)$ & 0.319 \\
\hline Blood pressure $\geq 140 / 90 \mathrm{mmHg}, \mathrm{n}(\%)$ & $134(42)$ & $85(39)$ & $49(48)$ & 0.134 & $78(44)$ & $56(40)$ & 0.461 \\
\hline Fasting glucose $\geq 126$ mg/dl, n (\%) & $92(29)$ & $60(28)$ & $32(31)$ & 0.494 & $48(27)$ & $44(31)$ & 0.407 \\
\hline Total cholesterol $\geq 240 \mathrm{mg} / \mathrm{dl}, \mathrm{n}(\%)$ & $45(14)$ & $32(15)$ & $13(13)$ & 0.632 & $18(10)$ & $27(19)$ & $0.021^{*}$ \\
\hline
\end{tabular}

*Statistically significant result 
141 seropositive $(9.39 \pm 2.64)$ than in the 178 seronegative $(8.74 \pm 2.61)$ individuals $(p=0.021)$.

A fully-adjusted logistic regression model showed a significant (and independent) association between social risk and SARS-CoV-2 positivity (OR 1.15; 95\% CI 1.04-1.27; $p=0.008$ ). For every unit of the total SDH score, the odds of SARS-CoV-2 seropositivity increased $15 \%$ (95\% CI $3.7-27 \%)$. The single covariate remaining significant in this model was total high cholesterol levels, and poor physical activity achieve a marginal association (Table 2). In addition, logistic regression models were fitted to assess the independent association between each of the five individual

Table 2 Fully-adjusted logistic regression model showing the independent association between the social determinants of health and SARS-CoV-2 seropositivity (as the dependent variable)

\begin{tabular}{llll}
\hline SARS-CoV-2 seropositivity & Odds ratio & $95 \%$ CI & $p$ value \\
\hline Social determinants of health & 1.15 & $1.04-1.27$ & $0.008^{*}$ \\
Age & 0.98 & $0.94-1.01$ & 0.161 \\
Female gender & 0.96 & $0.54-1.71$ & 0.893 \\
Primary school education & 1.49 & $0.84-2.64$ & 0.169 \\
Heavy alcohol intake & 0.99 & $0.46-2.13$ & 0.977 \\
Current smoker & 0.63 & $0.17-2.32$ & 0.487 \\
Body mass index $\geq 30 \mathrm{~kg} / \mathrm{m}^{2}$ & 1.09 & $0.61-1.94$ & 0.771 \\
Poor physical activity & 0.35 & $0.12-1.04$ & 0.060 \\
Poor diet & 0.47 & $0.13-1.65$ & 0.239 \\
Blood pressure $\geq 140 / 90 \mathrm{mmHg}$ & 0.93 & $0.57-1.51$ & 0.763 \\
Fasting glucose $\geq 126 \mathrm{mg} / \mathrm{dl}$ & 0.99 & $0.59-1.68$ & 0.981 \\
Total cholesterol $\geq 240 \mathrm{mg} / \mathrm{dl}$ & 2.09 & $1.07-4.08$ & $0.031^{*}$ \\
\hline
\end{tabular}

*Statistically significant result components of SDH and SARS-CoV-2 positivity, after adjusting for the above-mentioned covariates (Table 3 ). In these models, housing $(p=0.007)$ and family situation $(p=0.047)$ were the individual components of SDH significantly associated with SARS-CoV-2 seropositivity. However, the latter was not associated with SARS-CoV-2 seropositivity in the univariate model and its association in multivariate analysis was markedly influenced by age. As seen in Table 3, the Odds ratio of SDH components were all above 1 , and therefore they contributed to the main score of SDH to be significantly associated with SARS-CoV-2 seropositivity.

\section{Discussion}

This study shows a robust and independent association between SDH (and some of their individual components) and SARS-CoV-2 seropositivity. The individual component of SDH more strongly associated with SARS-CoV-2 seropositivity was housing, which suggested that lack of basic home facilities, i.e., absence of a flushing toilet and closed sewage disposal system, may increase the risk of acquisition of SARS-CoV-2 infection.

The above-mentioned editorial comments-worried about the importance of SDH on the risk of SARS-CoV-2 acquisition and spread-dealt with problems facing by developed societies [8-10]. In such settings, race/ethnicity, low scholarity, living in poor neighborhoods, use of public transportation, low income, less frequent access to healthy foods and disparities in access to medical care, have been argued as major responsibles for the social inequities that

Table 3 Univariate analyses and logistic regression models (adjusted for all covariates) showing associations between individual components of social determinants of health, and SARS-CoV-2 seropositivity

\begin{tabular}{|c|c|c|c|c|c|}
\hline & Total series $(\mathrm{n}=319)$ & Seronegative $(\mathrm{n}=178)$ & Seropositive $(\mathrm{n}=141)$ & $\begin{array}{l}\text { Significance } \\
\text { (univariate) }\end{array}$ & $\begin{array}{l}\text { Significance (multi- } \\
\text { variate models) }\end{array}$ \\
\hline Family situation & $1.66 \pm 1.11$ & $1.57 \pm 1.04$ & $1.77 \pm 1.18$ & 0.109 & $\begin{array}{l}\text { OR } 1.25 \\
(95 \% \text { CI } 1-1.57) \\
p=0.047^{*}\end{array}$ \\
\hline Economic status & $1.97 \pm 0.93$ & $3.09 \pm 0.99$ & $3.27 \pm 1.18$ & 0.086 & $\begin{array}{l}\text { OR } 1.23 \\
(95 \% \text { CI } 0.95-1.59) \\
p=0.111\end{array}$ \\
\hline Housing & $1.74 \pm 0.79$ & $1.63 \pm 0.78$ & $1.89 \pm 0.78$ & $0.003 *$ & $\begin{array}{l}\text { OR } 1.52 \\
(95 \% \text { CI } 1.12-2.06) \\
p=0.007 *\end{array}$ \\
\hline Social relationships & $1.35 \pm 0.85$ & $1.37 \pm 0.86$ & $1.33 \pm 0.83$ & 0.676 & $\begin{array}{l}\text { OR } 1.03 \\
(95 \% \text { CI } 0.76-1.39) \\
p=0.847\end{array}$ \\
\hline Support networks & $1.11 \pm 0.44$ & $1.08 \pm 0.29$ & $1.15 \pm 0.57$ & 0.156 & $\begin{array}{l}\text { OR } 1.67 \\
95 \% \text { CI } 0.90-3.09 \\
p=0.102\end{array}$ \\
\hline
\end{tabular}

*Statistically significant result 


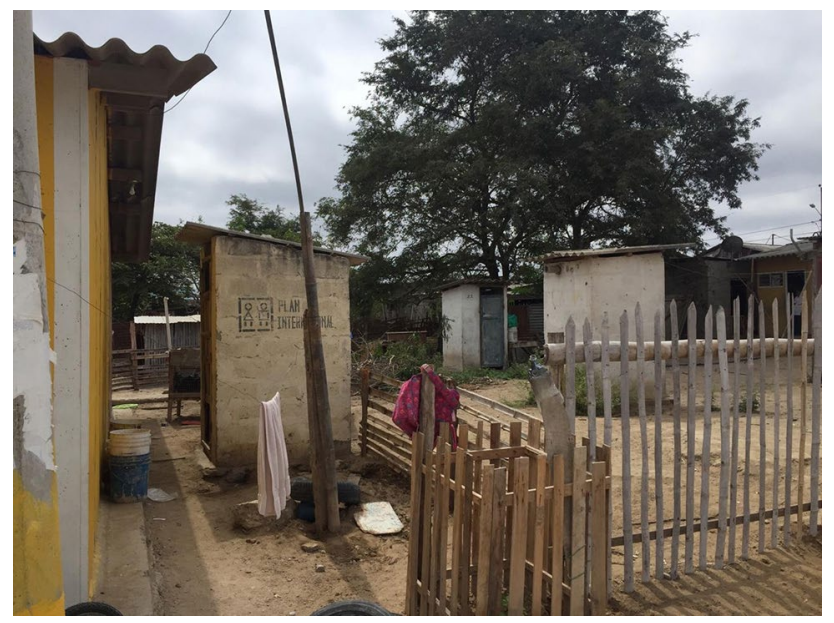

Fig. 1 Backyards of several houses in Atahualpa showing open latrines due to lack of in-house flushing toilet systems

may favor SARS-CoV-2 acquisition. To these, it must be added some other more basic needs that may put individuals living in underserved rural communities at particular risk of acquiring transmissible diseases, such as SARS-CoV-2 and other infections [5].

In Atahualpa, lack of flushing toilets lead to the use of open latrines for feces disposal, and this contributed to the poor housing scores achieved by many participants of this study (Fig. 1). It has been suggested that the fecal-oral route may contribute to the spread of SARS-CoV-2 infection in rural villages of the developing world [5], on the basis of studies showing the presence of this virus in human feces [20]. Our study provides indirect support to this hypothesis. The use of open latrines is often associated with poor hygienic habits and inadequate handling of human feces, which may facilitate fecal-oral transmission of the disease. Interestingly, these behaviors are not related to economic income or education among Atahualpa residents, as demonstrated in the present study where scholarity or wages were not associated with SARS-CoV-2 seropositivity.

To the best of our knowledge, no study has systematically addressed the role of SDH in SARS-CoV-2 acquisition and spread in rural communities. This is an important novel aspect of this study, as it shows that rural populations of Latin America are additionally burdened by poor SDH and do not seem to be prepared for this pandemic at all. This provides further support to previous fears of mass dissemination of the disease in these settings [21-23].

Besides its novelty, a major strength of this study is the high coverage and unbiased inclusion of long-term participants in the Atahualpa Project cohort, in whom several risk factors and conditions have been well characterized $[12,13]$. Nevertheless, the study has limitations. The study population was limited to older adults, and we missed the role of
SDH in the infection status of younger villagers. Individuals recently infected would have tested negative because they could be in the process of building their antibody response. In addition, while the test we used is reported to have high reliability, we cannot rule out a small degree of misclassification due to false positive or false negative results.

Knowledge on the burden of social risk and its association with SARS-CoV-2 infection is indispensable for public health planning and the development of cost-effective preventive strategies for controlling modifiable factors that are in the path of SARS-CoV-2 acquisition and spread among older adults living in underserved communities, where public health resources are already stretched to the limits.

Funding This study was supported by an unrestricted grant from Universidad Espiritu Santo - Ecuador, Samborondón, Ecuador.

\section{Compliance with Ethical Standards}

Conflict of interest The authors declare that they have no conflicts of interest.

\section{References}

1. Retrieved July 2, 2020, from https://www.worldometers.info/coron avirus/.

2. Zhao, Z., Li, X., Liu, F., Zhu, C., Ma, C., \& Wang, L. (2020). Prediction of the COVID-19 spread in African countries and implications for prevention and control: A case study in South Africa, Egypt, Algeria, Nigeria, Senegal and Kenia. The Science of the Total Environment, 729, 138959.

3. Caicedo-Ochoa, Y., Rebellón-Sánchez, D. E., Peñaloza-Rallón, M., Cortés-Motta, H. F., \& Méndez-Fandiño, Y. R. (2020). Effective reproductive number estimation for initial stage of COVID-19 pandemic in Latin American countries. International Journal of Infectious Diseases, 95, 316-318.

4. Cimerman, S., Chebado, A., Arns-da-Cunha, C., \& RodríguezMorales, A. J. (2020). Deep impact of COVID-19 in the healthcare of Latin America: The case of Brazil. The Brazilian Journal of Infectious Diseases, 24(2), 93-95.

5. Miller, M. J., Loaiza, J. R., Takyar, A., \& Gilman, R. H. (2020). COVID-19 in Latin America: Novel transmission dynamics for a global pandemic? PLoS Neglected Tropical Diseases, 14, e0008265.

6. Meneses-Navarro, S., Freyermuth-Enciso, M. G., PelcastreVillafuerte, B. E., Campos-Navarro, R., Meléndez-Navarro, D. M., \& Gómez-Flores-Ramos, L. (2020). The challenges facing indigenous communities in Latin America as they confront the COVID-19 pandemic. The International Journal for Equity in Health, 19(1), 63.

7. Burki, T. (2020). COVID-19 in Latin America. The Lancet Infectious Diseases, 20(5), 547-548.

8. Turner-Musa, J., Ajayi, O., \& Kemp, L. (2020). Examining social determinants of health, stigma, and COVID-19 disparities. Healthcare (Basel), 8(2), E168.

9. Abrams, E. M., \& Szefler, S. J. (2020). COVID-19 and the impact of social determinants of health. The Lancet. https://doi. org/10.1016/S2213-2600(20)30234-4. 
10. Rollston, R., \& Galea, S. (2020). COVID-19 and the social determinants of health. American Journal of Health Promotion, 34(6), 687-689.

11. Del Brutto, O. H., Mera, R. M., Del Brutto, V. J., Costa, A. F., $\&$ Recalde, B. Y. (2020). Cerebrovascular correlates of dementia in community-dwelling older adults living in rural communities-The Three Villages Study. Rationale and protocol of a population-based prospective cohort study. Journal of Stroke \& Cerebrovascular Diseases, 29(4), 104656.

12. Del Brutto, O. H., Dong, C., Rundek, T., Elkind, M. S., Del Brutto, V. J., \& Sacco, R. L. (2013). Cardiovascular health status among Caribbean Hispanics living in Northern Manhattan and Ecuadorian natives/mestizos in rural coastal Ecuador: A comparative study. Journal of Community Health, 38(4), 634-641.

13. Del Brutto, O. H., Mera, R. M., Peralta, L. D., et al. (2020). Cardiovascular health status among community-dwelling Ecuadorian natives living in neighboring rural communities: The three villages study. Journal of Community Health, 45(1), 154-160.

14. Del Brutto, O. H., Castillo, P. R., Sedler, M. J., et al. (2018). Reasons for declining consent in a population-based cohort study conducted in a rural South American Community. Journal of Environmental and Public Health, 2018, 8267948.

15. Cabrera González, D., Menéndez Caicoya, A., Fernández Sánchez, A., et al. (1999). Evaluación de la fiabilidad y validez de una escala de valoración social en el adulto. Atención Primaria, 23(7), 434-440.

16. Cao, Y., Liu, X., Xiong, L., \& Cai, K. (2020). Imaging and clinical features of patients with 2019 novel coronavirus SARS-CoV-2: A systematic review and meta-analysis. Journal of Medical Virology. https://doi.org/10.1002/jmv.15822.

17. Lloyd-Jones, D., Hong, Y., Labarthe, D., et al. (2010). Defining and setting national goals for cardiovascular health promotion. The American Heart Association's strategic impact goal through 2020 and beyond. Circulation, 121(4), 586-613.

18. Wang, Y., Tian, H., Zhang, L., et al. (2020). Reduction of secondary transmission of SARS-CoV-2 in households by face mask use, disinfection and social distancing: A cohort study in Beijing, China. British Medical Journal Global Health, 5(5), e002794.

19. Sethuraman, N., Jeremiah, S. S., \& Ryo, A. (2020). Interpreting diagnostic tests for SARS-CoV-2. JAMA. https://doi.org/10.1001/ jama.2020.8259.

20. Xiao, F., Sun, J., Xu, Y., et al. (2020). Infectious SARS-CoV-2 in feces of patients with severe COVID-19. Emerging Infectious Diseases. https://doi.org/10.3201/eid2608.200681.

21. Amigo, I. (2020). Indigenous communities in Brazil fear pandemic's impact. Science, 368(6489), 352.

22. Ferrante, L., \& Fearnside, P. M. (2020). Protect indigenous peoples from COVID-19. Science, 368(6488), 251.

23. Rascombe, P. (2020). Rural areas at risk during COVID-19 pandemic. Lancet Infectious Diseases, 20(5), 545.

Publisher's Note Springer Nature remains neutral with regard to jurisdictional claims in published maps and institutional affiliations. 